\title{
Cluster of Paediatric Severe Pneumonia Caused by Streptococcus pneumoniae Serotype 3 in Fully Vaccinated Children in Luxembourg
}

\author{
Julie Verbeke ${ }^{1,2}$, Bushra Zucca ${ }^{1,3}$, Valerie Flaum ${ }^{6}$, Paul Philipe ${ }^{6}$, \\ Chantal Tsobo ${ }^{4}$, Alexandre Mzabi ${ }^{5}$, Monique Perrin ${ }^{5}$, Armand Biver ${ }^{1}$, \\ Meriem Mastouri ${ }^{1}$ and Isabel de La Fuente Garcia ${ }^{1 *}$ \\ ${ }^{1}$ Department of Pediatrics, National Center for Pediatrics, Centre Hospitalier de \\ Luxembourg, Luxembourg \\ ${ }^{2}$ Department of Pediatrics, University Hospital of Louvain-la-Neuve, Belgium \\ ${ }^{3}$ Department of Pediatrics, University Hospital of Brussels, Belgium \\ ${ }^{4}$ Microbiology Department, Centre Hospitalier de Luxembourg, Luxembourg \\ ${ }^{5}$ Department of Microbiology, National Health Laboratory, Luxembourg \\ ${ }^{6}$ Department of Paediatric Surgery, National Center for Pediatrics, Centre \\ Hospitalier de Luxembourg, Luxembourg \\ *Corresponding Author: Isabel de La Fuente Garcia, Department of Pediatrics, \\ National Center for Pediatrics, Centre Hospitalier de Luxembourg, Luxembourg.
}

Received: November 01, 2021

Published: November 24, 2021

(C) All rights are reserved by Isabel de La

Fuente Garcia., et al.

\section{Abstract}

Streptococcus pneumoniae is the first cause of bacterial pneumonia in children. Since the implementation of routine immunization in 2004, the incidence of severe pneumococcal infections has decreased in Luxembourg. Thirteen-valent conjugated vaccine (PVC13), used routinely since 2010 , is the only licensed pneumococcal conjugated vaccine with serotype 3 polysaccharide in its formulation. The incidence rate of empyema in Luxembourg was 5/100.000 person-years (children aged 0 to 6 years old) between 2014 and 2019, representing an annual average of 2.2 admissions in our National Paediatric center.

We report a cluster of 6 cases of complicated pneumonia (empyema, necrosis, and abscess) in otherwise healthy children hospitalized between December 2019 and February 2020 (9 weeks period). Their median age was 4 years (1-5). All of them had been vaccinated with PCV13 according to the national vaccination schedule in Luxembourg. All patients were treated with intravenous antibiotics. Four patients were transiently admitted to the intensive care unit. Two patients underwent video-assisted thoracoscopic surgery to debride the pleural space. All patients recovered from their pneumonia. Pneumococcal pneumonia was confirmed in five patients (positive PCR on pleural fluid) with serotyping being performed in four of them and identifying ser3 pneumococcus.

We present a cluster of cases of complicated pneumococcal pneumonia caused by ser3 pneumococcus in children vaccinated with PCV13. Several other European countries have reported cases of complicated ser3 pneumococcal pneumonia in previously vaccinated children, indicating the need for close monitoring of this serotype and development of better vaccination strategies against ser3 pneumococcus.

Keywords: Pneumococcal Pneumonia; Complicated Pneumonia; Streptococcus pneumoniae Serotype 3; Pneumococcal Conjugated Vaccine; Vaccine Failure

\section{Abbreviations}

CPS: Capsular Polysaccharide; CRP: C-reactive Protein; CT: Computed Tomography; Hb: Haemoglobin; IPD: Invasive Pneumococ- cal Disease; IV: Intravenous; PCR: Polymerase Chain Reaction; PCV: Pneumococcal Conjugated Vaccine; PCV7: Seven-valent Pneumococcal Conjugated Vaccine; PCV13: Thirteen-valent Pneumococcal

Citation: Isabel de La Fuente Garcia., et al. "Cluster of Paediatric Severe Pneumonia Caused by Streptococcus pneumoniae Serotype 3 in Fully Vaccinated Children in Luxembourg". Acta Scientific Microbiology 4.12 (2021): 67-74. 
Conjugated Vaccine; PICU: Paediatric Intensive Care Unit; SIADH: Syndrome of Inappropriate Anti-diuretic Hormone Secretion; $S$. pneumoniae: Streptococcus pneumoniae; ser: Serotype; ser3 pneumococcus: Streptococcus pneumoniae Serotype 3; US: Ultrasonography; WBC: White Blood Cells

\section{Introduction}

Streptococcus pneumoniae (S. pneumoniae) is the leading cause of bacterial pneumonia in children. Complicated pneumonia is characterized by empyema, lung necrosis or abscess.

In Luxembourg, the 7-valent conjugated vaccine (PCV7, covering the serotypes $4,6 \mathrm{~B}, 9 \mathrm{~V}, 14,18 \mathrm{C}, 19 \mathrm{~F}$ and $23 \mathrm{~F}$ ) is used routinely since 2004 . It was replaced by the 13 -valent conjugated vaccine (PCV13, covering the serotypes included in PCV7 plus serotypes 1, $3,5,6 \mathrm{~A}, 7 \mathrm{~F}$ and $19 \mathrm{~A}$ ) in 2010. Since 2011, the national vaccination schedule counts 3 doses $(2+1$, with injections at 2,4 and 12 months). The vaccination coverage is excellent with $95.8 \%$ in 2018 [1].

Since the systematic vaccination against $S$. pneumoniae in the early 2000's in several European countries with conjugated pneumococcal vaccines, the overall incidence of invasive pneumococcal disease (IPD: sepsis, meningitis, and severe pneumonia) decreased, in both vaccinated and unvaccinated children (herd immunity). However, we observed a shift to non-vaccinal pneumococcal serotype strains after PCV implementation. After the implementation of PCV7, the main serotypes responsible of IPD in children were ser1, 3 and 19A. The use of PCV13 decreased the incidence of overall IPD and IPD due to vaccine included serotypes, but we also observed an increasing incidence of pneumococcal infections due to nonvaccine serotypes [2].

Between 2014 and 2019, the incidence rate of hospitalization for empyema due to any pathogen in our National Pediatric Centre was 5/100.000 children-year (aged from 0 to 6 years), representing 2.2 annual admissions.

\section{Cases Presentation}

\section{Case 1}

A 7-year-old male was admitted to the Pediatric Emergency Department on December $27^{\text {th }} 2019$ with a history of five-day fever, cough and abdominal pain. He was otherwise healthy and fully vaccinated according to the national schedule. He was born full term in Luxembourg; his parents were French and Luxembourgish.
His physical examination at admission revealed an asymmetric auscultation with posterior lower right lobe hypoventilation. The patient complained about thoracic pain in decubitus position and at deep inspiration.

Initial routine laboratory testing revealed an inflammatory syndrome, with a C-reactive protein (CRP) level reaching $237 \mathrm{mg} / \mathrm{L}$, a white blood cell (WBC) count of $16.1 \times 10 * 9 / \mathrm{L}$. Hb, platelet count, renal function were all normal, ionogram showed a minor hyponatremia.

The chest radiograph showed an opacification of the inferior right lung lobe, with a moderate pleural effusion (Figure 1).

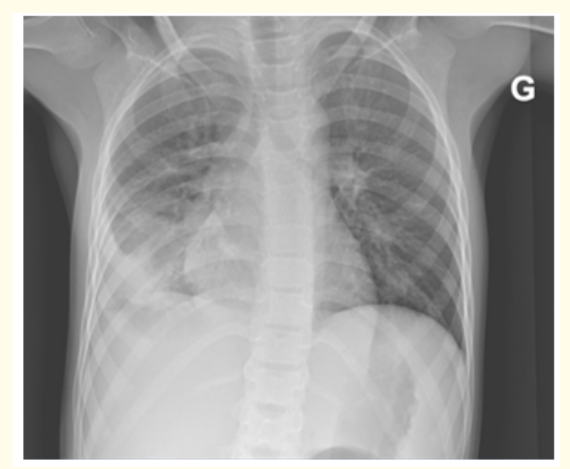

Figure 1: Chest radiograph at the admission showing inferior right lung consolidation associated with pleural effusion.

The patient was hospitalized, and antibiotics were immediately started with intravenous (IV) Amoxicillin (200 mg/kg/d) and oral Azithromycin (10 mg/kg/d the first day then $5 \mathrm{mg} / \mathrm{kg} / \mathrm{d}$ ), according to the local protocol. After two days, the patient was still febrile, his laboratory testing showed a CRP level of $250 \mathrm{mg} / \mathrm{L}$ and a WBC count of $12.92 \times 10^{*} 9 / \mathrm{L}$ despite the treatment. The chest radiograph showed the same opacification, with a worsened homolateral effusion. A chest ultrasonography (US) showed right lung densification with liquefaction zones (maximum $12 \times 8 \times 4 \mathrm{~mm}$ ), associated with an-echogenic septate effusion, with an axe of $16 \mathrm{~mm}$ maximum in axillar topography. The child was transferred in the pediatric intensive care unit (PICU), and underwent thoracoscopy and tube placement for the empyema. The treatment was replaced by IV Ceftriaxone $(100 \mathrm{mg} / \mathrm{kg} / \mathrm{d})$ and IV Clindamycin $(40 \mathrm{mg} /$ $\mathrm{kg} / \mathrm{d}$ ). He did not require ventilatory support nor oxygen therapy.

The pleural fluid analysis revealed an exudate (proteins 49.1 $\mathrm{g} / \mathrm{l}$ ), with a WBC count of $1480 / \mu \mathrm{L}$ (54\% polymorphonuclear neu- 
trophils). The pleural fluid culture and hemocultures remained sterile, but a PCR performed on pleural fluid was positive for $S$. pneumoniae. The serotyping performed on this strain at our national reference laboratory identified ser3.

The fever stopped quickly after the tube placement which could be removed after five days. After 10 days the patient was discharged from hospital. He continued 5 days of oral antibiotic therapy (Amoxicillin $100 \mathrm{mg} / \mathrm{kg} / \mathrm{d}$ ) for a total of fifteen days. The patient was followed in the respiratory and surgery outpatient clinic. His immunologic work-up realized after the acute phase was normal (Immunoglobulins G, M, and A dosage, vaccinal antibody responses (tetanus, diphtheria, and pneumococcus), absence of Howell Jolly bodies at blood smear and complement dosage (C3, C4, CH50)). At 6-months follow-up, he was healthy with no clinical signs of sequelae and a chest radiograph showed a blunting of the right costophrenic angle and right pleural thickening.

\section{Case 2}

A 22-month-old male was admitted to the Paediatric Emergency Department on January 5th 2020 with a history of 3-day fever and cough. He was otherwise healthy and fully vaccinated according to the national schedule, the last dose of PCV13 made in 2018. He was born premature (35 weeks) in Luxembourg with no neonatal event. His parents were originated from Bosnia and Montenegro. He attended day care.

His physical examination at the admission revealed an asymmetric auscultation with hypoventilation in the right upper lobe.

Initial routine laboratory testing revealed an inflammatory syndrome, with a CRP level reaching $470 \mathrm{mg} / \mathrm{L}$, a WBC of $21.1 \mathrm{x}$ $10 * 9 / \mathrm{L}$. Hb was low $(9.6 \mathrm{~g} / \mathrm{dl})$. He had hyponatremia with $\mathrm{Na}$ of $133 \mathrm{mmol} / \mathrm{L}$. Platelet count and renal function were normal.

The chest radiograph showed an opacification of the upper right lung lobe, associated with consolidation of the medium right lobe, probably due to parapneumonic atelectasis (Figure 2a).

The patient was hospitalized, and we started the treatment with IV Amoxicillin (200 mg/kg/d) and oral Azithromycin $(10 \mathrm{mg} / \mathrm{kg} / \mathrm{d}$ the first day then $5 \mathrm{mg} / \mathrm{kg} / \mathrm{d}$ ). After 3 days, the patient was not recovering despite an appropriate treatment with persistent fever and laboratory testing showing a CRP level of $330 \mathrm{mg} / \mathrm{L}$ and a WBC of $15.48 \times 10^{*} 9 / \mathrm{L}$. The chest radiograph showed a pleural effu- sion associated to the right upper lobe opacification with necrosis zones (Figure 2b), and a chest US showed right lung densification, associated with echogenic septate effusion in pleural space with a maximum axe of $9 \mathrm{~mm}$. He was transferred in the PICU, underwent thoracoscopy and two tubes placement to drain the empyema. During video-assisted thoracoscopy, an abscess of the lung parenchyma was confirmed. The treatment was shifted to IV Ceftriaxon (100 $\mathrm{mg} / \mathrm{kg} / \mathrm{d}$ ) and IV Clindamycin (40 mg/kg/d). He did not require ventilatory support nor oxygen therapy.

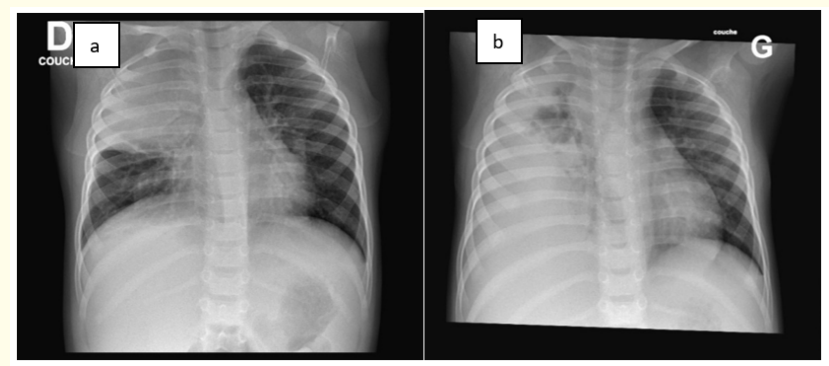

Figure 2: (a) Chest radiograph at the admission showing opacification of the upper right lobe associated with medium right lobe atelectasis; (b) Chest radiograph after two days, showing major pleural effusion and necrosis zone inside the opacification.

The pleural fluid culture and haemocultures remained sterile, but a PCR performed on pleural fluid was positive for ser3 pneumococcus.

The fever stopped after a total of 13 days of treatment. The pleural tube was removed after 4 days. After the surgery and the tube placement, haemoglobin level decreased to a minimum of $7.4 \mathrm{~g} / \mathrm{dl}$, requiring a transfusion of packed red blood cells. The patient was discharged after 17 days, and the oral treatment (Amoxicillin 100 $\mathrm{mg} / \mathrm{kg} / \mathrm{d}$ ) was continued for 11 days, for a total of 28 days. The patient was followed in the respiratory outpatient clinic. His immunologically work-up (as per case 1) came back normal. At 6-month follow-up, he was healthy with no clinical signs of sequelae and a chest radiograph showed minor upper right lobe atelectasis.

\section{Case 3}

A 5-year-old girl was admitted to the Paediatric Emergency Department on January 12th 2020 for cough and vomiting. She initially consulted 9 days earlier in another centre for cough and 
fever and was prescribed antibiotics (Amoxicillin/clavulanate) for bronchitis, without further work-up. She took her medicine only two days, and the fever stopped after six days.

She was otherwise healthy and fully vaccinated according to the national schedule, with 3 doses of PCV13. She was born full term in Luxembourg; her parents were originated from Kosovo and Montenegro. She is the cousin of case 2 .

Her physical examination at the admission was normal, except an impaired general condition.

Laboratory testing showed moderate inflammatory syndrome, with a CRP level of $100 \mathrm{mg} / \mathrm{L}$ and a WBC of $10.5 \times 10^{*}$ / $/ \mathrm{L}$.

The chest radiograph showed a round upper right lobe consolidation, suggesting an abscess (Figure 3).

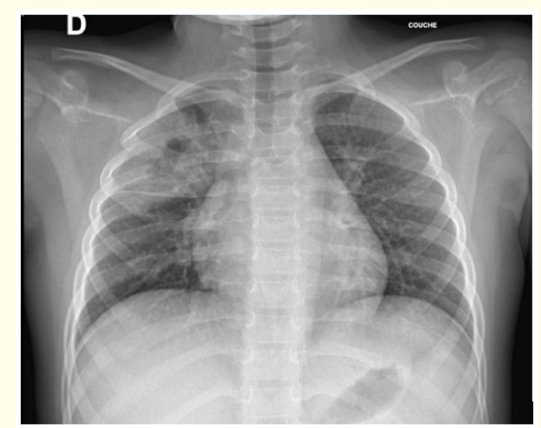

Figure 3: Chest radiograph at the admission, showing round upper right lobe opacification containing aeric zones.

The girl was hospitalized, and we changed treatment to IV antibiotics, with Amoxicillin/clavulanate $(100 \mathrm{mg} / \mathrm{kg} / \mathrm{d})$. On the second day of hospitalisation, a chest ultrasonography showed right posterior upper lobe consolidation with hydro-aeric bright zones in it, corresponding to the abscess, with no associated effusion. Thus, the antibiotic was shifted to IV Ceftriaxone $(200 \mathrm{mg} / \mathrm{kg} / \mathrm{d})$ and IV Clindamycin ( $40 \mathrm{mg} / \mathrm{kg} / \mathrm{d})$. She never had fever during her stay. She was discharged after3 days, and continued IV antibiotics for 6 days, then oral antibiotics (Amoxicillin $100 \mathrm{mg} / \mathrm{kg} / \mathrm{d}$ ) for 10 days. She had a total of 19 days of antibiotics.

The haemoculture remained sterile. We did not obtain pleural liquid as there was no pleural effusion, and there was no need to drain the abscess.
The patient was followed by her paediatrician and saw the infectious diseases consultant once at 2-month follow-up. She was clinically normal with no sequelae and a normal chest radiograph. Her immunologic work-up (as per case 1) came back normal.

\section{Case 4}

A 3-year-old male was admitted to the Paediatric Emergency Department on January 29th 2020 with a history of 6-day fever, abdominal pain, cough and impaired general condition. He was otherwise healthy and fully vaccinated according to the national schedule, the last PCV13 dose made in 2017. He was born at full term in Luxembourg, his parents were Portuguese and Luxembourgish. He was going to day care.

His physical examination on admission revealed an asymmetric auscultation with left lung crackles and hypoventilation.

Initial routine laboratory testing revealed an inflammatory syndrome, with a CRP level of $310 \mathrm{mg} / \mathrm{L}$, a WBC of $21.8 \times 10 * 9 / \mathrm{L}$. He had hyponatremia with a natrium level of $133 \mathrm{mmol} / \mathrm{l}$. Renal function and the rest of ionogram were normal.

The chest radiograph showed an opacification of the middle-left lung lobe associated with moderate homolateral pleural effusion.

The patient was hospitalized, and antibiotics were started with IV Amoxicillin (200 mg/kg/d) and oral Azithromycin $(10 \mathrm{mg} / \mathrm{kg} / \mathrm{d}$ the first day then $5 \mathrm{mg} / \mathrm{kg} / \mathrm{d}$ ), according to the local protocol. After three days, we controlled a chest radiograph, which showed worsening of the effusion. A chest US showed left lung densification, associated with major purulent effusion in pleural space, pushing the lung to the right and deviating the mediastinum. A chest CT was performed and showed necrotizing pneumonia of the left lung associated with abscess and empyema (Figure 4). His laboratory testing showed a CRP level of $267 \mathrm{mg} / \mathrm{L}$ and a WBC of $36.1 \times 10^{* 9} / \mathrm{L}$. The child was transferred in the PICU and underwent tube placement for the empyema. The antibiotics were shifted to IV Ceftriaxone (100 mg/kg/d) and IV Clindamycin (40 mg/kg/d). He required oxygen therapy for 24 hours.

The pleural fluid culture and haemocultures remained sterile, but a PCR performed on pleural fluid was positive for ser3 pneumococcus.

Citation: Isabel de La Fuente Garcia., et al. "Cluster of Paediatric Severe Pneumonia Caused by Streptococcus pneumoniae Serotype 3 in Fully Vaccinated Children in Luxembourg". Acta Scientific Microbiology 4.12 (2021): 67-74. 


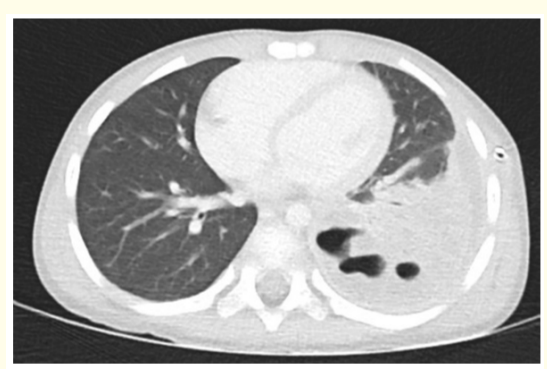

Figure 4: CT showing necrotizing pneumonia in the left lung associated with abscess and empyema.

The fever stopped after a total of 12 days. The pleural tube was removed after 5 days. The patient was discharged after 12 days, and the oral treatment (Amoxicillin $100 \mathrm{mg} / \mathrm{kg} / \mathrm{d}$ ) was continued for 21 days, for a total of 33 days. The patient was followed in the respiratory outpatient clinic. His immunological work-up (as per case 1) came back normal l. At 6-month follow-up, he was healthy with no clinical signs of sequelae and a normal chest radiograph.

\section{Case 5}

An 18-month-old male was admitted to the Paediatric Emergency Department on February 11th 2020 with a history of 4-day fever, cough and impaired general condition. He was otherwise healthy and fully vaccinated according to the national schedule, with 3 doses of PCV13. He was born full term in Luxembourg; his parents were Dutch and Luxembourgish.

His physical examination at the admission revealed an asymmetric auscultation with left lung hypoventilation, and moderate respiratory distress (tachypnoea, retractions, nose flaring).

Initial routine laboratory testing revealed an inflammatory syndrome, with a CRP level of $380 \mathrm{mg} / \mathrm{L}$, a WBC of $17.7 \times 10^{*} \mathrm{~g} / \mathrm{L}$, and a hyponatremia with Natrium level of $130 \mathrm{mmol} / \mathrm{l}$. The renal function and platelet count were normal.

The chest radiograph showed multiple opacifications of the inferior left lung lobe with parapneumonic effusion. A chest US confirmed the pleural effusion, with septa, suggesting purulent nature of the pleural fluid (Figure 5a).

The patient was hospitalized, and antibiotics were immediately started with IV Ceftriaxone (100 mg/kg/d) and IV Clindamycin (40 $\mathrm{mg} / \mathrm{kg} / \mathrm{d}$ ). The US made after 24 hours showed worsening of the empyema and necrosis zones, justifying tube placement. He did not require PICU admission, ventilatory support nor oxygen therapy.

The pleural fluid culture and haemocultures remained sterile, but a PCR performed on pleural fluid was positive for ser3 pneumococcus.

After 7 days, another chest US was made as the patient was still painful and showed necrosis of the left inferior lobe. The fever stopped after eight days of antibiotic therapy. The pleural tube was removed after seven days. He had inflammatory and post-operatory anaemia ( $\mathrm{Hb} 8.6 \mathrm{~g} / \mathrm{dl}$ ) which did not require transfusion. The patient was discharged after 14 days, and the IV antibiotic was continued for 4 days. The oral treatment (Amoxicillin $100 \mathrm{mg} / \mathrm{kg} / \mathrm{d}$ ) was continued for 14 days, for a total of 33 days. The patient was followed in the respiratory outpatient clinic. His immunological work-up (as per case 1) came back normal. At 6-months follow-up, he was healthy with no clinical signs of sequelae and a chest radiograph showed left pleural thickening (Figure 5b).

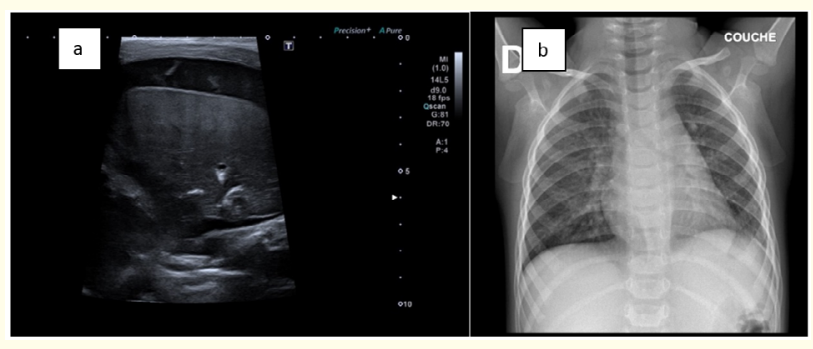

Figure 5: (a) Chest US showing septate major pleural effusion and lung condensation; (b) Chest radiograph at 6-months follow-up, showing left pleural thickening.

\section{Case 6}

A 4-year-old male was admitted to the Paediatric Emergency Department on February 28th 2020 with a history of 8-day fever, cough and impaired general condition. He was otherwise healthy and fully vaccinated according to the Belgian national schedule, with 3 doses of PCV13. He was born at 37 weeks in Belgium, his parents were Belgian. He was going to school in Belgium.

His physical examination at the admission revealed an asymmetric auscultation with left lung hypoventilation and respiratory distress (tachypnoea, retractions). 
Initial routine laboratory testing revealed an inflammatory syndrome, with a CRP level reaching $292 \mathrm{mg} / \mathrm{L}$, a WBC of $11.7 \mathrm{x}$ $10 * 9 / \mathrm{L}$. The remaining of the laboratory testing was normal.

The chest radiograph showed opacification of the whole left lung except apical zone (Figure 6).

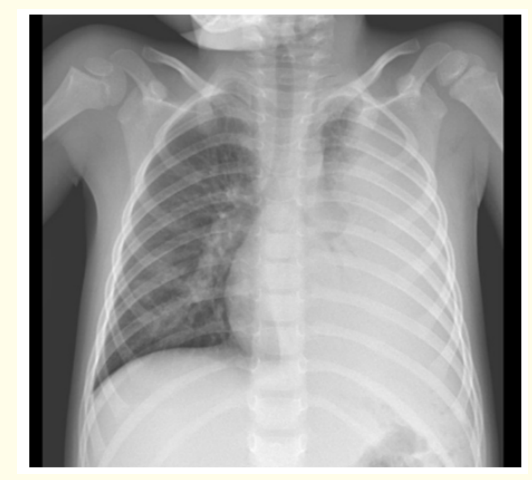

Figure 6: Chest radiograph at the admission, showing almost whole left hemithorax opacification.

The patient was hospitalized immediately in the PICU, and antibiotics were started with IV Ceftriaxone $(100 \mathrm{mg} / \mathrm{kg} / \mathrm{d})$, oral Azithromycin (10 mg/kg/d one day) and IV Clindamycin (40 mg/ $\mathrm{kg} / \mathrm{d}$ ). After 3 days, the patient was still febrile, and the chest US showed consolidation of the whole left lung with necrosis in the upper lobe, and major effusion in pleural space (maximum $27 \mathrm{~mm}$ in inferior zone). He required oxygen therapy with high flow nasal cannula for 4 days.

The pleural fluid analysis showed an exudate and a WBC count of $3400 / \mu \mathrm{L}$. Pleural fluid culture and haemocultures remained sterile, but a PCR performed on pleural fluid was positive for $S$. pneumoniae. Unfortunately, serotyping of the strain was inconclusive (insufficient bacterial load in the pleural fluid to obtain a serotyping result).

The pleural tube was removed after 3 days. The patient was discharged after 11 days, and oral treatment (Amoxicillin $100 \mathrm{mg}$ / $\mathrm{kg} / \mathrm{d}$ ) was continued for 21 days, for a total of 32 days. The fever stopped after 7 days of treatment. The patient was followed in the respiratory outpatient clinic. His immunological workout (as per case 1) came back normal. At 3-months follow-up, he was healthy with no clinical signs of sequelae and the chest radiograph showed blunting of the left costophrenic angle.

\section{Discussion}

PCVs are highly effective in preventing IPD, with only $2 \%$ due to PVCs failure [3]. Since the routine use of PCV13, we observed an overall decreased incidence of S. pneumonia infections in several European countries $[2,4,5]$. However, the effectiveness of PCV13 against ser3 has been repeatedly questioned these last years.

PVC13 is well tolerated and showed efficacy in a randomized double-blind trial which enabled its licencing in 2010. However, this study comparing PCV7 to PVC13 immunogenic response showed that ser3 was the less immunogenic serotype contained in PCV13, providing the threshold level of specific IgG $\geq 0.35 \mu \mathrm{g} / \mathrm{ml}$ in only $88 \%$ of fully vaccinated participants and showing the lowest mean specific IgG level [6]. A 2014 post-licensure indirect cohort study in England, Wales and Ireland suggests that the $0.35 \mu \mathrm{g} / \mathrm{mL}$ threshold underestimates the true correlate of protection in PCV13 specific serotypes, the needed IgG specific level to provide a significant protection against ser3 being much higher $(2.83 \mu \mathrm{g} / \mathrm{ml})$. According to this hypothesis, the estimated effectiveness of PCV13 against ser3 would be only $26 \%$ [7]. Moreover, the synthesis of capsular polysaccharide (CPS) during growth in ser3 could explain the lower efficacy of anti-type 3 CPS antibody-mediated protection [8]. This rapid production of CPS would also lead to a reduced humoral immune response, explaining the higher rates of necrotizing pneumonia and empyema associated with ser3 [9]. Regarding other IPD, PCV13 showed efficacy against septicaemia and meningitis due to ser3 [10].

Six cases of complicated pneumococcal pneumonia (empyema, abscess, or necrosis) are described in this report (Table 1). They occurred between December 2019 and February 2020 (9-week period). The patient's median age was 4 years (1-5). They were all healthy with no medical nor surgical history, and all of them had been fully vaccinated with PCV13 according to the national vaccination schedule in Luxembourg $(2+1)$. Their main symptoms were fever, abdominal pain, cough, and impaired general condition. They all had inflammatory syndrome and hyperleukocytosis on admission laboratory testing. Four of them needed PICU admission, five had pleural tube placement (median length of 5 days), two underwent video-assisted thoracoscopic surgery to debride the pleural space and two needed oxygen therapy. The identification of S. pneumoniae was made in all cases by real-time PCR (lytA gene target) on pleural fluid, except for case 3 . The serotyping was made in all cases by multiplex PCR (CDC protocol) on pleural fluid, except for case 6 . As case 3 was related to and in contact with case 2 , we suggested 
that she had been infected by the same serotype. The other patients were not related and were all going to different schools or day care centres. The four first cases were initially treated according to local protocol with IV Amoxicillin and oral Azithromycin, then, seeing the evolution of previous cases of the cluster, the two last cases were immediately treated with second-line antibiotics (IV Ceftriaxone and IV Clindamycin). As pneumococcus identification was only performed through PCR without growth on standard culture, no antibiotic sensitivity testing was available for any of the cases. Most of them had hyponatremia linked to parapneumonic syndrome of inappropriate anti-diuretic hormone secretion (SIADH), which was treated by fluid intake restriction. Two had anaemia, probably due to inflammation and tube placement, and one required transfusion. They all fully recovered after 15 to 33 days of antibiotics (median length of thirty days). Their median hospitalisation length was 11.5 days. Their follow-up chest radiograph was normal except pleural thickening in some cases. Their immunological work-up (Immunoglobulins G, M, and A dosage, vaccinal antibody responses (tetanus, diphteria and pneumococcus), absence of Howell Jolly bodies at blood smear and complement dosage (C3, C4, CH50)) came back normal.

\begin{tabular}{|c|c|c|c|c|c|c|}
\hline & Case 1 & Case 2 & $\begin{array}{c}\text { Case } \\
3\end{array}$ & Case 4 & Case 5 & $\begin{array}{c}\text { Case } \\
6\end{array}$ \\
\hline Sex & M & M & $\mathrm{F}$ & M & M & M \\
\hline Age (years) & 5 & $1 \frac{1 / 2}{2}$ & 5 & 3 & $11 / 2$ & 4 \\
\hline PCV13 (doses) & 3 & 3 & 3 & 3 & 3 & 3 \\
\hline $\begin{array}{l}\text { CRP at } \\
\text { admission } \\
(\mathrm{mg} / \mathrm{L})\end{array}$ & 237 & 470 & 100 & 310 & 380 & 292 \\
\hline Complication & $\mathrm{E}+\mathrm{A}+\mathrm{N}$ & $\mathrm{E}+\mathrm{A}+\mathrm{N}$ & A & $\mathrm{E}+\mathrm{N}$ & $\mathrm{E}+\mathrm{N}$ & $\begin{array}{c}\mathrm{E}+ \\
\mathrm{N}\end{array}$ \\
\hline $\begin{array}{l}\text { Days with } \\
\text { chest tube }\end{array}$ & 5 & 4 & 0 & 5 & 7 & 3 \\
\hline Thoracoscopy & + & + & - & - & - & - \\
\hline $\begin{array}{l}\text { PICU } \\
\text { admission }\end{array}$ & + & + & - & + & - & + \\
\hline Oxygen & - & - & - & + & - & + \\
\hline $\begin{array}{l}\text { Length of IV } \\
\text { antibiotherapy } \\
\text { (days) }\end{array}$ & 10 & 17 & 9 & 12 & 19 & 11 \\
\hline $\begin{array}{l}\text { Length of total } \\
\text { antibiotherapy } \\
\text { (days) }\end{array}$ & 15 & 28 & 19 & 33 & 33 & 32 \\
\hline Identification & PCR + ser3 & PCR + ser3 & No & PCR + ser 3 & PCR + ser3 & \begin{tabular}{|l|l|} 
& PCR \\
\end{tabular} \\
\hline
\end{tabular}

Table 1: Characteristics of children who developed complicated pneumonia due to $S$. pneumoniae serotype 3.

E: Empyema; A: Abscess; N: Necrosis.
Several European countries (Portugal [11], Spain [12], Greece [13], Italy [10], Great Britain [14] and Germany [15]) reported complicated pneumonia due to ser3 pneumococcus, despite a complete PCV13 vaccination according to the local schedule $2+1$ or $3+1$ schedules) in children without any comorbidities. This is the first report from Luxembourg showing similar data. Our data, combined with the previous studies reporting complicated pneumococcal pneumonia due to ser3 pneumococcus in fully vaccinated children, illustrates reduced protection by PCV13 against ser3. A candidate 15 -valent pneumococcal conjugated vaccine, containing serotypes included in PCV13 plus serotypes 22F and 33F, shows promising results considering its immunogenicity against ser3, with higher specific IgG levels, and achieving the threshold of specific IgG $\geq 0.35$ $\mu \mathrm{g} / \mathrm{mL}$ in more patients [16]. Continuous monitoring and clinical reports of pneumococcal serotypes in IPD, in particular of ser3, are of major importance in order to adapt and improve vaccination strategies against pneumococcal disease.

Our last case occurred in February 2020, and we observed a last case of complicated pneumonia due to another serotype in the beginning of March 2020. We can suggest this small epidemic stopped at that specific time due to the COVID-19 pandemic and the containment policies, as mentioned in a recent European study including Luxembourgish data [17].

\section{Conclusion}

We report a cluster of pediatric complicated pneumonia due to ser3 pneumococcus despite full vaccination according to the Luxembourg schedule in otherwise healthy children. This report, combined with previous European studies, suggests a lack of protection by current pneumococcal vaccines against ser3, and indicates the need for close monitoring and larger studies to evaluate the protection afforded by PCVs against ser3 complicated pneumonia.

\section{Acknowledgements}

We thanks to Dr Van Nieuwenhuyse, Dr Gauthiez and Dr Vieira for the contribution of this work (radiology team).

\section{Conflict of Interest}

We have no conflict of interest to declare.

\section{Bibliography}

1. Pivot D and Leite S. «Enquête de couverture vaccinale 2018 au Grand-Duché de Luxembourg». Le Gouvernement du Grand-Duché de Luxembourg (2018). 
2. Waight P., et al. "Effect of the 13-valent pneumococcal conjugate vaccine on invasive pneumococcal disease in England and Wales 4 years after its introduction: an observational cohort study". Lancet Infectious Diseases 15 (2015): 535-543.

3. Oligbu G., et al. "Pneumococcal conjugate vaccine failure in children: A systematic review of the literature". Vaccine 34.50 (2016): 6126-6132.

4. Madhi F, et al. "Change in Bacterial Causes of Community-Acquired Parapneumonic Effusion and Pleural Empyema in Children 6 Years After 13-Valent Pneumococcal Conjugate Vaccine Implementation". Journal of the Pediatric Infectious Diseases Society 8.5 (2019): 474-477.

5. Azzari C., et al. "Significant impact of pneumococcal conjugate vaccination on pediatric parapneumonic effusion: Italy 20062018". Vaccine 37.20 (2019): 2704-2711.

6. Snape M., et al. "Immunogenicity and Reactogenicity of a 13-valent-pneumococcal conjugated vaccine administered at 2, 4, and 12 months of age". The Pediatric Infectious Disease Journal 12 (2010): 80-90.

7. Andrews N., et al. "Serotype-specific effectiveness and correlates of protection for the 13-valent pneumococcal conjugate vaccine: a postlicensure indirect cochort study". Lancet Infectious Diseases 14.9 (2014): 839-846.

8. Choi EH., et al. "Capsular Polysaccharide (CPS) Release by Serotype 3 Pneumococcal Strains Reduces the Protective Effect of Anti-Type 3 CPS Antibodies". Clinical and Vaccine Immunology 23.2 (2016): 162-167.

9. Bender J., et al. "Pneumococcal necrotizing pneumonia in Utah: does serotype matter?" Clinical Infectious Diseases 46 (2008): 1346-1352.

10. Lodi L., et al. "Impact of the 13-Valent Pneumococcal Conjugate Vaccine on Severe Invasive Disease Caused by Serotype 3 Streptococcus pneumoniae in Italian Children". Vaccine 7 (2019): 128.

11. Silva-Costa C., et al. "Pediatric Complicated Pneumonia caused by Streptococcus pneumoniae serotype 3 in 13-valent pneumococcal conjugated vaccinees, Portugal, 2010-2015". Emerging Infectious Disease 24.7 (2018): 1307-1314.

12. Moraga Llop F., et al. "Vaccine failures in patient properly vaccinated with 13-valent pneumococcal conjugate vaccine in Catalonia, a region with low vaccination coverage". The Pediatric Infectious Disease Journal 35 (2016): 460-463.
13. Antachopoulos C., et al. "Parapneumonic pleural effusions caused by Streptococcus pneumoniae serotype 3 in children immunized with 13-valent conjugated pneumococcal vaccine". The Pediatric Infectious Disease Journal 33 (2014): 81-83.

14. Oligbu G., et al. "Characteristics and serotype distribution of childhood cases of invasive pneumococcal disease following pneumonoccal conjugated vaccination in England et Wales, 2006-2014". Clinical Infectious Disease 65.7 (2017): 11911198.

15. Goettler D., et al. "Increase in Streptococcus pneumoniae serotype 3 associated parapneumonic pleural effusion/empyema after the introduction of PCV13 in Germany". Vaccine 38.3 (2020): 570-577.

16. Greenberg D., et al. "Safety and immunogenicity of 15-valent pneumococcal conjugate vaccine (PCV15) in healthy infants". Vaccine 36.45 (2018): 6883-6891.

17. Brueggemann A., et al. "Changes in the incidence of invasive disease due to Streptococcus pneumoniae, Haemophilus influenzae, and Neisseria meningitidis during the COVID-19 pandemic in 26 countries and territories in the Invasive Respiratory Infection Surveillance Initiative: a prospective analysis of surveillance data". Lancet Digit Health 3 (2021): e360-370.

\section{Volume 4 Issue 12 December 2021 (C) All rights are reserved by Isabel de La Fuente Garcia., et al.}

